\title{
Estimating Cascading Failure Risk with Random Chemistry
}

\author{
Pooya Rezaei, Student Member, IEEE, Paul D. H. Hines, Senior Member, IEEE, Margaret J. Eppstein
}

\begin{abstract}
The potential for cascading failure in power systems adds substantially to overall reliability risk. Monte Carlo sampling can be used with a power system model to estimate this impact, but doing so is computationally expensive. This paper presents a new approach to estimating the risk of large cascading blackouts triggered by multiple contingencies. The method uses a search algorithm (Random Chemistry) to identify blackoutcausing contingencies, and then combines the results with outage probabilities to estimate overall risk. Comparing this approach with Monte Carlo sampling for two test cases (the IEEE RTS-96 and a 2383 bus model of the Polish system) illustrates that the new approach is at least two orders of magnitude faster than Monte Carlo, without introducing measurable bias. Moreover, the approach enables one to compute the sensitivity of overall blackout risk to individual component-failure probabilities in the initiating contingency, allowing one to quickly identify low-cost strategies for reducing risk. By computing the sensitivity of risk to individual initial outage probabilities for the Polish system, we found that reducing three line-outage probabilities by $50 \%$ would reduce cascading failure risk by $33 \%$. Finally, we used the method to estimate changes in risk as a function of load. Surprisingly, this calculation illustrates that risk can sometimes decrease as load increases.
\end{abstract}

Index Terms-Cascading failure, Monte Carlo sampling, power systems reliability

\section{INTRODUCTION}

Large blackouts are low-probability, high-impact events; i.e., they happen infrequently, but when they do happen, they can lead to catastrophic social and economic effects, such as the North American blackouts of Aug. 2003 [1] and Sept. 2011 [2]. For this reason cascading failure risk assessment is increasingly required by reliability standards (e.g., [3]) and is a focus of IEEE Power and Energy Society activities [4].

A primary goal of reliability risk analysis methods is to produce statistical reliability measures, such as Expected Energy Not Supplied (EENS), that allow one to compare different versions of a particular system, or to evaluate changes in a system over time. The science and practice of Monte Carlo (MC) methods for power system reliability studies are well established [5], particularly for evaluating generation and transmission adequacy. However, for some types of problems, Monte Carlo methods can require many samples to produce high-confidence statistical results, causing the approach to be computationally prohibitive or even infeasible in some cases

This work was supported in part by U.S. Dept. of Energy award $\neq$ deoe0000447 and by U.S. National Science Foundation Award \#ECCS-1254549.

The authors are with the College of Engineering and Mathematical Sciences, University of Vermont, Burlington, VT (e-mail: \{pooya.rezaei,paul.hines,maggie.eppstein\}@uvm.edu).
[6]. Some studies reduce the computational effort due to sampling by: 1) variance reduction techniques [7|, 2) state-space pruning [8], 3) parallel and distributed computation [6], 4) pseudo-sequential simulation [9], or 5) Artificial Neural Networks [10]. Generally, the results provide small improvements (typically less than a factor of 10 speedup). To our knowledge, [10] describes an approach with the most substantial speedup: a factor of 45 over standard Monte Carlo.

Methods for applying sampling techniques to cascading failure risk estimation are less well established [4], for several reasons. First, the simulation of cascading failures remains a difficult problem, making it difficult to estimate the eventual impact (typically measured in the amount of lost load) of a cascade-triggering disturbance. As a result, standard power system reliability models tend to capture only one or two outage generations, ignoring the impact of subsequent outages [11]. Second, even if cascading sizes can be accurately estimated, those sizes can be at any scale: from a few MW to tens of $\mathrm{GW}$. The nature of cascading gives rise to the well-documented power-law in blackout sizes [12], [13]. This implies that the occurrence probabilities of particular combinations of outages (and operator errors) that could trigger large cascading failures are very low, making it necessary to observe several representative events to obtain an accurate risk estimate [14]. Third, the size of the search space of all possible $n-k$ contingencies that might trigger a cascade, where $n$ is the number of components that might fail and $k$ is the number that did fail, is enormous and grows exponentially with $n$ and $k$. This further increases the need for a large number of simulations.

Despite these challenges, there are a number of studies focused on the modeling of cascading failures (e.g., [11], [15][17]). A smaller number of papers have adapted sampling techniques to the problem of cascading failure risk estimation [11], [18]. In doing so, some have used variance reduction techniques to reduce the computational effort [19]-[22], which led to a speedup factor of 5-10 in [19], and 2-4 in [22]. Non-sampling approaches, such as branching process models [23], [24], can provide efficient estimates of risk, but abstract away some details, such as the ability to compute the relative contributions of particular outages to overall risk.

In our prior work [25], we adapted a search algorithm, known as Random Chemistry (RC), to the problem of finding large collections of $n-k$ contingencies that lead to cascading failure. However, this initial work did not explain how the approach could be used for risk estimation. In this paper, we derive a method to use the Random Chemistry algorithm to estimate blackout risk due to cascading failure, given a 
few simplifying assumptions, and compare the computational efficiency of our approach with that of Monte Carlo sampling. This paper builds on preliminary results reported in [26] and [27], extending our method to compute the relative impacts of individual components on overall risk and showing how increasing load and generator dispatch method impact risk.

The remainder of the paper is organized as follows. Section II defines cascading failure risk and introduces two general approaches to estimate risk. Section III describes our method for using Random Chemistry for cascading failure risk estimation, and section IV presents simulation results. Finally, section $\mathrm{V}$ provides our conclusions.

\section{TWO APPROACHES TO RISK ESTIMATION}

A standard measure of risk due to a random disturbance is the product of event probability and its severity (or cost) [4]. If $S(\mathcal{C}, x)$ is a measure of the severity of the events proceeding from an arbitrary disturbance $\mathcal{C}$ to a system at a particular state $x$, then the risk due to $\mathcal{C}$ is:

$$
R(\mathcal{C}, x)=\operatorname{Pr}(\mathcal{C}) S(\mathcal{C}, x)
$$

If we denote the set of all possible disturbances by $\Omega$, then the system risk is:

$$
R(x)=\sum_{\forall c \in \Omega} R(c, x)=\sum_{\forall c \in \Omega} \operatorname{Pr}(c) S(c, x)
$$

In this paper, $\mathcal{C}$ denotes a random disturbance, while $c$ (the lower case) denotes an individual disturbance. Therefore, one can think of $S(\mathcal{C}, x)$ as a random variable that maps a random event $\mathcal{C}$ to its severity, and interpret $R(x)$ as $E[S(\mathcal{C}, x)]$, i.e., the expected value of $S(\mathcal{C}, x)$.

Since it may not be feasible to compute the severity of all disturbances for a system, one approach for estimating system risk is to estimate $R(x)$ from a smaller sample of all possible disturbances. One option is Monte Carlo sampling, in which one draws randomly from $\Omega$ based on the probability function $\operatorname{Pr}(\mathcal{C})$. The average severity then converges to the true risk, given a sufficiently large set of samples:

$$
\hat{R}_{M C}(x)=\frac{1}{\left|\Omega_{a}\right|} \sum_{c \in \Omega_{a}} S(c, x)
$$

where $\hat{R}$ is an estimated value of $R$ in this and subsequent notations, $\Omega_{a}$ is a set that results from sampling randomly from $\Omega$, and $\left|\Omega_{a}\right|$ is the number of elements in $\Omega_{a}$. Note that $\Omega_{a}$ may include duplicates and the empty set, depending on the sampling approach.

A key problem with this approach is that there are many events for which $S(c, x)=0$, which means that Monte Carlo will spend a lot of time sampling from events that have no impact on $R(x)$. Alternatively, if one could sample exclusively from the subspace $\Omega_{B}$ such that $S(c, x)>0, \forall c \in \Omega_{B}$, and we somehow knew the size of this subset, then risk could be more efficiently estimated by computing:

$$
\hat{R}(x)=\frac{\left|\Omega_{B}\right|}{\left|\Omega_{b}\right|} \sum_{c \in \Omega_{b}} \operatorname{Pr}(c) S(c, x)
$$

where $\Omega_{b}$ is a representative subset sampled from $\Omega_{B}$, i.e., $\Omega_{b} \subseteq \Omega_{B}$. Clearly, (4) converges to (2) as $\Omega_{b} \rightarrow \Omega_{B}$. In order to avoid a biased outcome when $\left|\Omega_{b}\right| \ll\left|\Omega_{B}\right|, \Omega_{b}$ needs to be an unbiased sample, such that it provides a representative sample of $\Omega_{B}$ across a range of event sizes and probabilities. The idea in (4) is somewhat similar to importance sampling [28].

In this paper, we develop a method for estimating cascading blackout risk in power systems based on (4), and compare the convergence speed with Monte Carlo sampling in (3).

\section{MethoD}

The specific focus of this paper is estimating risk due to cascading failures triggered by exogenously-caused branch (transmission line or transformer) outage contingencies. In this case, each contingency $c \in \Omega$ is a subset of the branches in the grid ( $c \subseteq\{1, \ldots, n\}$ ), and $\Omega$ is the set of all possible branch outage combinations. While there are other types of contingencies that might trigger cascades, branch outages provide a useful starting point. Adapting the method to incorporate other type of contingencies (e.g., generator outages) is trivial. In this paper, we assume that the exogenously-caused branch outages are statistically independent, such that for two single contingencies $c_{i}$ and $c_{j}, \operatorname{Pr}\left(c_{i} \cap c_{j}\right)=\operatorname{Pr}\left(c_{i}\right) \operatorname{Pr}\left(c_{j}\right)$. While there are methods in the literature for modeling common mode outages (e.g., [29]) and other types of correlations (e.g., [30]), the risk from these correlations is not captured in the results presented here. Verifying the efficiency of the Random Chemistry risk estimation method for the case of correlated outages and other types of contingencies remains for future work.

In this paper, $S(c, x)$ represents the output of a simulator that gives blackout sizes in MW, resulting from any initiating contingency $c$ applied to the system with state $x$ such that $0 \leq S(c, x) \leq S_{0}$, where $S_{0}$ is the total load in the system. In general, Monte Carlo sampling can quickly estimate $R(x)$ for blackouts of small sizes. However, because of the heavytailed statistics of cascading failures, estimating the risk due to larger blackouts is more difficult. Here, we focus on computing the risk resulting from blackouts larger than some fraction $0<\alpha<1$ of $S_{0}$. Specifically, we study the risk of blackouts $5 \%$ and larger, i.e., for $S(\mathcal{C}, x) \geq 0.05 S_{0}$. In order to do so, we replace $S(\mathcal{C}, x)$ in 3 with $S_{\alpha}(\mathcal{C}, x)$, where:

$$
S_{\alpha}(\mathcal{C}, x)=\left\{\begin{array}{cc}
S(\mathcal{C}, x) & S(\mathcal{C}, x) \geq \alpha S_{0} \\
0 & \text { otherwise }
\end{array}\right.
$$

\section{A. Risk from minimal contingencies}

A unique aspect of the Random Chemistry algorithm (see Appendix I and [25]) is that it is designed to find "minimal" blackout-causing contingencies. A minimal blackout-causing contingency is a set of outages that result in a large blackout (in this paper we define large as $\geq 5 \%$ ), but would not result in a large blackout if any one of the outages in the multiple-contingency had not occurred. For brevity we refer to disturbances of this sort as malignancies. Formally, a set of outages $d$ is a malignancy if $S(d, x) \geq 0.05 S_{0}$ and 
$\nexists c^{\prime} \in \Omega: c^{\prime} \subset d \wedge S\left(c^{\prime}, x\right) \geq 0.05 S_{0}$. In order to use the data from Random Chemistry, we need to be able to estimate the risk from all $n-k$ contingencies, including the non-minimal ones, from data about malignancies. To do so, we make the following assumption:

Assumption 1: Given a malignancy $d$, which triggers a blackout of size $S(d, x)$, the blackout sizes triggered by all supersets of $d$ can be estimated by the size of blackout triggered by d, i.e., $\forall c^{\prime \prime} \supset d \rightarrow S\left(c^{\prime \prime}, x\right)=S(d, x)$.

Clearly, Assumption 1 does not hold for every case. There are some superset contingencies that trigger smaller blackouts and some supersets that cause larger blackouts. In Appendix II, we evaluate Assumption 1, comparing the actual blackout sizes of all $n-3$ supersets with the values that are estimated by minimal $n-2$ subsets for the Polish system (described in section IV. We find that Assumption 1 causes the $n-3$ risk estimate to be $2.5 \%$ higher than the actual value for $n-3$ risk. Given that the $n-3$ risk is $24.0 \%$ of the total risk, the total impact of Assumption 1 on the overall risk estimate is to increase it by only $0.6 \%$.

As one adds more components to a minimal malignancy, making larger supersets, the blackout size difference between the two events becomes larger, but because the probability of high order events $(n-k$ for $k \geq 4)$ is orders of magnitude lower than probability of its malignancy subset, the overall impact becomes much smaller, and at some point negligible for high order events.

This section shows, by a small example, how to use Assumption 1 to estimate risk efficiently using the minimal contingency data that Random Chemistry provides.

Let us assume that in a small system with 5 transmission lines, Random Chemistry has found an $n-3$ malignancy, $d_{1}=\{1,2,3\}$. (By definition, this means that none of the $n-2$ subset contingencies, $c_{1}^{\prime}=\{1,2\}, c_{2}^{\prime}=\{1,3\}$ and $c_{3}^{\prime}=\{2,3\}$, cause a $5 \%$ or larger blackout.) Using our previous assumption that line failures are independent, the exact risk associated with $d_{1}$ and all its supersets is:

$$
\begin{aligned}
R\left(\hat{d}_{1}, x\right)= & S\left(d_{1}, x\right) p_{1} p_{2} p_{3}\left(1-p_{4}\right)\left(1-p_{5}\right)+ \\
& S\left(c_{1}^{\prime \prime}, x\right) p_{1} p_{2} p_{3} p_{4}\left(1-p_{5}\right)+ \\
& S\left(c_{2}^{\prime \prime}, x\right) p_{1} p_{2} p_{3}\left(1-p_{4}\right) p_{5}+ \\
& S\left(c_{3}^{\prime \prime}, x\right) p_{1} p_{2} p_{3} p_{4} p_{5}
\end{aligned}
$$

where $\hat{d}_{1}$ denotes $d_{1}$ together with all its supersets: $c_{1}^{\prime \prime}=$ $\{1,2,3,4\}, c_{2}^{\prime \prime}=\{1,2,3,5\}, c_{3}^{\prime \prime}=\{1,2,3,4,5\}$, and $p_{i}$ is the line-failure probability for line $i$. Given Assumption 1, $S\left(d_{1}, x\right)=S\left(c_{i}^{\prime \prime}, x\right), \forall i \in\{1,2,3\}$, which means that:

$$
\begin{aligned}
& R\left(\hat{d}_{1}, x\right)=S\left(d_{1}, x\right) p_{1} p_{2} p_{3}\left[\left(1-p_{4}\right)\left(1-p_{5}\right)+\right. \\
&\left.p_{4}\left(1-p_{5}\right)+\left(1-p_{4}\right) p_{5}+p_{4} p_{5}\right]
\end{aligned}
$$

Expanding the term in the bracket shows that it equals 1, which means that:

$$
R\left(\hat{d}_{1}, x\right)=S\left(d_{1}, x\right) p_{1} p_{2} p_{3}=S\left(d_{1}, x\right) \operatorname{Pr}\left(\hat{d}_{1}\right)
$$

where $\operatorname{Pr}\left(\hat{d}_{1}\right)$ is the probability of the malignancy $d_{1}$ and all of its supersets, i.e., any event that includes at least the branches in $d_{1}$. A similar formulation holds for supersets of any malignancy. Therefore, Assumption 1 enables us to estimate risk of a malignancy and its supersets solely using the information provided by the malignancy itself. In general, for each malignancy $d$, we have:

$$
R(\hat{d}, x)=S(d, x) \operatorname{Pr}(\hat{d})=S(d, x)\left(\prod_{i \in d} p_{i}\right)
$$

\section{B. General Random Chemistry risk estimation}

Our method separately estimates the risk due to $n-2$ and $n-3$ malignancies. We use the symbol $\Omega_{m, k} \subset \Omega$ to be the set of all $n-k$ malignancies. After $i$ runs, Random Chemistry will have found a certain number of events from each $\Omega_{m, k}$ set. Let $i_{k}$ be the number of times that Random Chemistry has found a not-necessarily-unique member of $\Omega_{m, k}$ and $\Omega_{R C, k}$ be the set of unique members found. By tracking the rate at which unique members of $\Omega_{m, k}$ are found, if $i_{k}$ is sufficiently large, we can estimate the size of $\Omega_{m, k}$ (see section III-C). Let $\hat{m}_{k}$ be this estimate. Given this notation, and the derivation in (7), we can simultaneously capture the $n-k$ risk from a set of malignancies and all of their supersets based on (4) and (8), as follows:

$$
\hat{R}_{R C, k}(x)=\frac{\hat{m}_{k}}{\left|\Omega_{R C, k}\right|} \sum_{d \in \Omega_{R C, k}} S(d, x)\left(\prod_{i \in d} p_{i}\right)
$$

where $\hat{R}_{R C, k}(x)$ is the estimated $n-k$ blackout risk by Random Chemistry. The results in section IV] indicate that the sum of these values over $k$ gives a reasonable estimate of total blackout risk:

$$
\hat{R}_{R C}(x)=\sum_{k=2}^{k_{\max }} \hat{R}_{R C, k}(x)
$$

We have set $k_{\max }=5$ while running Random Chemistry previously, since larger multiple contingencies are highly improbable, and (at least for the case of uncorrelated outages) have a minuscule contribution to the total risk. However, the size of the $n-5$ and $n-4$ sets are so large that for realworld systems, it is not computationally practical to estimate $\hat{m}_{5}$ and $\hat{m}_{4}$. Thus we generally use $k_{\max }=3$ for risk calculations. Since this captures the risk from an increasingly large collection of $n-\{2,3\}$ malignancies and all of their supersets, the results appear to be highly representative of the true system risk (see section IV].

Note that there is a caveat in considering the malignancy supersets as in 97. Every contingency set causing a large blackout contains a malignancy, but this malignancy is not necessarily unique. The contingencies that contain more than one unique malignancy are considered more than once in (9). For example, if $\{1,2\}$ and $\{1,3\}$ were both malignancies in a system, the superset $\{1,2,3\}$ would be taken into account two times in 99; once in $S(\{1,2\}, x) p_{1} p_{2}$ and once in $S(\{1,3\}, x) p_{1} p_{3}$. However, the number of these kinds of supersets is normally much lower than the total number of supersets of any order, so the overall error that they cause is 
negligible. In this paper, we are mainly studying the $n-2$ and $n-3$ risk, so we counted the exact number of $n-3$ supersets that have been counted more than once in the Polish system. Our inspection indicated that out of the total of 1,562,760 $n-3$ supersets of $n-2$ malignancies, 21,771 supersets $(1.4 \%)$ included more than one malignancy subset, and thus were counted more than once. Note that the exact number of malignancies that will be counted more than once depends on the particular system. It is also noteworthy that each of the higher order supersets may include more malignancies than each of the lower order ones, however, as mentioned before, the higher order contingencies have orders of magnitude lower probabilities and thus have an infinitesimal impact on the overall risk.

It is important to note that we find, in section IV that Assumption 1 and the above caveat do not measurably change the risk estimation results relative to the Monte Carlo estimate, which computes risk based on the exact probability and blackout sizes of all contingencies.

\section{Estimating the Size of Each $n-k$ Collection}

Initially, Random Chemistry primarily finds malignancies that are unique (not found previously). However, as the number of identified malignancies increases, the algorithm finds duplicates more frequently. By measuring the rate at which the algorithm finds new unique contingencies of a given size $k$, one can estimate the total number of minimal dangerous $n-k$ contingencies that cause blackouts. There are a variety of ways to estimate the set-size $\left|\Omega_{m, k}\right|$ as Random Chemistry progresses, such that: $\lim _{i_{k} \rightarrow \infty} \hat{m}_{k}\left(i_{k}\right)=\left|\Omega_{m, k}\right|$. In [25], we suggested an approach based on measuring the rate at which the algorithm is finding new malignancies. In this paper, we use another approach based on the number of unique malignancies found vs. the number of Random Chemistry runs. To illustrate, consider a jar of $N$ balls numbered $1, \ldots, N$. If balls are removed from the jar one at a time with the same probability (and then replaced), one can mathematically show that the expected number of unique balls drawn after $i$ draws $\left(N_{i}\right)$ follows:

$$
N_{i}=N\left(1-e^{r i}\right)
$$

where $r=\ln \left(1-\frac{1}{N}\right)$. Based on this idea, we suggest an alternative exponential model, which was empirically found to be more accurate for our application. We found that the number of unique malignancies found by Random Chemistry is best represented by the Cumulative Distribution Function (CDF) of the exponential Weibull distribution. Specifically, the number of unique $n-k$ malignancies in the set $\Omega_{R C, k}$ after $i_{k}$ runs follows:

$$
\left|\Omega_{R C, k}\left(i_{k}\right)\right| \sim \hat{m}_{k}\left(1-e^{-\left(\frac{i_{k}}{\lambda}\right)^{\mu}}\right)^{\nu}
$$

The parameters $\lambda, \mu, \nu$ and $\hat{m}_{k}$ (the estimate of interest) can be found by nonlinear least-squares fitting to a sequence of data for $i_{k}$ and $\left|\Omega_{R C, k}\left(i_{k}\right)\right|$. Fig. 1 shows the results of this method, where the the sizes of $n-2$ and $n-3$ malignancy sets are estimated for the Polish system. The results shown for $n-2$ are for three separate runs of Random Chemistry searching for
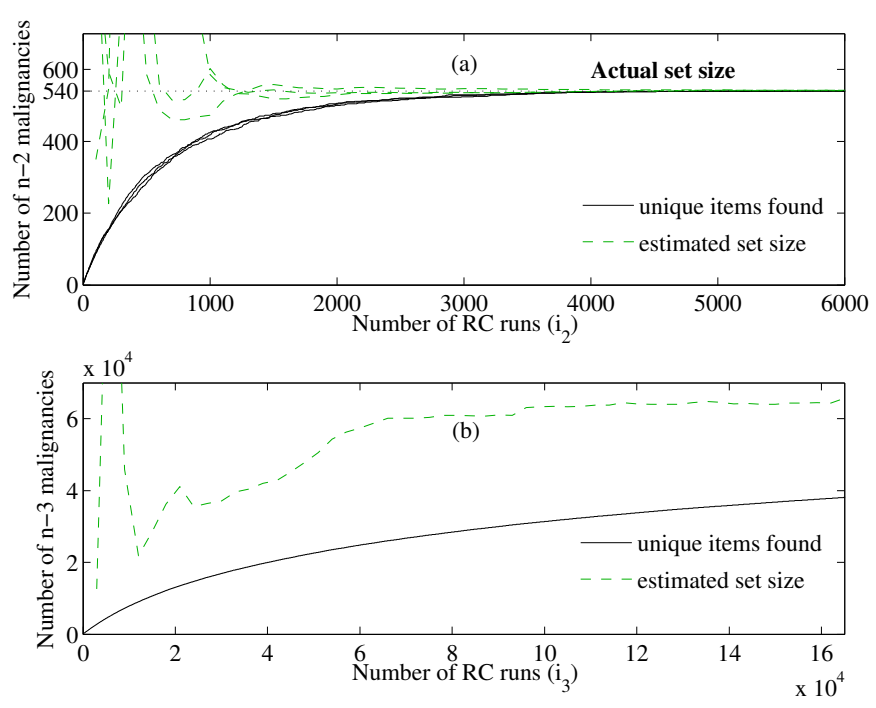

Figure 1. The number of unique (a) $n-2$, and (b) $n-3$ malignancies found $\left(\Omega_{R C, k}\right.$,solid line) and the estimate of the set size ( $\hat{m}_{k}$, dashed line) vs. the number of Random Chemistry runs that led to an $n-2\left(i_{2}\right)$ and $n-3\left(i_{3}\right)$ malignancy, respectively, in the Polish system.

malignancies in the Polish system (Fig.119). All three estimates converged to $\hat{m}_{k}=540$, which is the true number of $n-2$ malignancies, as verified by exhaustive search. Fig. 1p shows that the estimate of $\hat{m}_{3}$ is converging to about $6.4 \times 10^{4}$.

\section{Sensitivity of risk to component-failure probability}

As previously suggested, it is possible to use the Random Chemistry risk estimation approach in (9) to find the sensitivity of the overall risk to individual component-failure probability in the initiating contingency. To do so, we compute the partial derivative of $\hat{R}_{R C, k}(x)$ with respect to each branch failure probability $p_{i}$ and sum the result over $k \in\{2,3\}$ for all branches, as follows:

$$
\begin{gathered}
\frac{\partial \hat{R}_{R C, k}(x)}{\partial p_{i}}=\frac{\hat{m}_{k}}{\left|\Omega_{R C, k}\right|} \sum_{d \in \Omega_{R C, k}} S(d, x)\left(\prod_{\substack{j \in d \\
j \neq i}} p_{j}\right) \\
\frac{\partial \hat{R}_{R C}(x)}{\partial p_{i}}=\sum_{k=2}^{k_{\max }} \frac{\partial \hat{R}_{R C, k}(x)}{\partial p_{i}}
\end{gathered}
$$

This type of sensitivity analysis is particularly useful in finding critical components in a transmission system (see section IV-C3). Note that (14) provides the sensitivity of risk to individual outage probability in the initiating events, and to keep this paper focused, we do not study the failure probabilities when a cascade propagates. In future work, we will identify the lines that fail frequently during the course of cascades, and study the impact on risk of upgrading these lines.

\section{RESUlts}

We used two test systems to evaluate the proposed risk estimation method. The first is the 73-bus RTS-96, which 
has 120 branches and $8550 \mathrm{MW}$ of total load [29]. The second test system is a model of the 2004 winter peak Polish power system, which is available with MATPOWER [31]. This test system has 2896 branches (transmission lines and transformers), 2383 buses, and $24.6 \mathrm{GW}$ of total load. For the Polish case, some of the transmission lines were overloaded in the original system, so we increased line flow limits to be the larger of the existing limit and 1.05 times the precontingency line flows for each line, after increasing all loads by $10 \%$. We prepared the pre-contingency test cases for both systems using a preventive security-constrained dc optimal power flow (SCDCOPF) to make both cases $n-1$ secure with respect to branch outages (see [27] for details). The $n-1$ security constraints in the SCDCOPF model ensure that all flows remain less than or equal to the long-term emergency (LTE) rating after a single branch outage. The LTE rating is assumed to be $110 \%$ of the normal flow limit for all lines. The generation cost data, which is needed for the OPF, is not readily available for RTS-96. In this paper, we took the cost data from [32] based on the generation types in [29].

In both networks, we computed line failure probabilities from transmission line failure rates ( $\lambda$, outages/year), and assumed that each failure lasts for 1 hour on average (that the mean time to repair is 1 hour). Thus, the probability of line $i$ being in a non-working state during a particular sample is $p_{i}=\lambda_{i} / 8760$, where 8760 is the number of hours in a year. Note that this approximation is valid only for the case where $\lambda_{i} / 8760 \ll 1$, which is true, since all of the $\lambda$ in the test systems are approximately 1 . As the failure rate data were not available for the Polish system, we randomly assigned failure rates to transmission lines, such that the mean and variance of $\lambda$ 's were equal to those in the RTS-96.

\section{A. Simulator}

To test our method, we used an updated version of the cascading failure simulator used in [25]. In this simulator (known as DCSIMSEP) line flows are computed using a dc power flow calculation. When outages result in long-term emergency (LTE) rating violations (assumed to be $110 \%$ of the normal line flow limit), transmission lines trip in an amount of time that is proportional to the magnitude of the overload, such that more extreme overloads result in faster outages. The time delay is set such that if the line flow is $50 \%$ above the normal limit (that is the short-term emergency rating, STE), the line will trip in 5 seconds. When line outages result in the separation of a network into multiple connected components (islands), a combination of fast generator ramping, load shedding and generator tripping are used to restore a balance between supply and demand in each island. After each simulation, DCSIMSEP reports the number of line outages and the amount of load shedding that occurred. Illustrative video and source code for the simulator are available at [33].

DCSIMSEP does not describe several known mechanisms of cascading failure, such as voltage collapse, dynamic instability or hidden failures in protection systems. However, the risk analysis process proposed here can be used in combination with any simulator that reports the blackout size, given an initiating contingency. Future work will test the impact of these additional mechanisms on the efficiency of the proposed approach.

\section{B. Comparing Random Chemistry approach to Monte Carlo}

We applied the proposed method to our two test cases, and estimated large blackout risk as a function of total computational effort. These results were compared to results from a standard Monte Carlo simulation. The number of calls to DCSIMSEP is used as our measure of computational effort, because cascading failure simulation takes up the vast majority of risk estimation run-time. Actual run-time is not used explicitly since it depends on many factors, including the extent to which code is optimized. Each cascading failure simulation (by a call to DCSIMSEP) in the risk estimation process takes 0.3 to 0.5 seconds on average for the Polish system 1 , depending on the number of subsequent cascades. In our Monte Carlo implementation, a contingency is simulated only if the number of elements in the sampled contingency is 2 or larger, since the system was initially $n-1$ secure. While each Monte Carlo run needs either zero or one simulation, each successful Random Chemistry run requires approximately 47 simulations (when averaged over both successful and unsuccessful runs) on the Polish system.

Figs. 2a and 2p show the Monte Carlo and Random Chemistry risk convergence for the RTS-96 and the Polish systems, respectively. More specifically, Fig. 2 a shows the convergence of $E\left[S_{0.05}(\mathcal{C}, x)\right]$ for the RTS-96, while Fig. 2p shows the convergence of $E\left[S_{0.05}(\mathcal{C}, x)\right]$ and $E\left[S_{0.4}(\mathcal{C}, x)\right]$ for the Polish system. In the case of RTS-96, the largest blackout caused by $n-2$ and $n-3$ malignancies was less than $20 \%$ of total load, therefore we only explored $E\left[S_{0.05}(\mathcal{C}, x)\right]$.

Fig. 2 shows that the Random Chemistry approach is more than two orders of magnitude faster than Monte Carlo for both test systems. We also observe that Monte Carlo and Random Chemistry are converging to similar values, providing evidence that Assumption 1 does not measurably bias the results.

In addition, Table I compares the convergence numerically for $S \geq 5 \%$ in the Polish system. We computed the mean, variance and $95 \%$ confidence intervals (the range between $2.5^{\text {th }}$ and $97.5^{\text {th }}$ percentiles) for both Random Chemistry and Monte Carlo by bootstrapping, where random samples were taken with replacement from the original data. Results are for 1000 resampling trials, where for each trial, the number of samples were chosen for both Random Chemistry and Monte Carlo such that they required the same number of calls to the cascading failure simulator.

Table II shows that after $10^{6}$ calls to the simulator, the confidence interval for Monte Carlo is much wider than for Random Chemistry. Note that the very minor drift in the Random Chemistry confidence interval from $10^{6}$ to $2 \times 10^{7}$ calls to the simulator is due to the improved estimate of $\hat{m}_{3}$ (Fig. 1p).

\footnotetext{
${ }^{1}$ On a $2.66 \mathrm{GHz}$ Intel Core i7 MacBook Pro with $8 \mathrm{~GB}$ of memory
} 

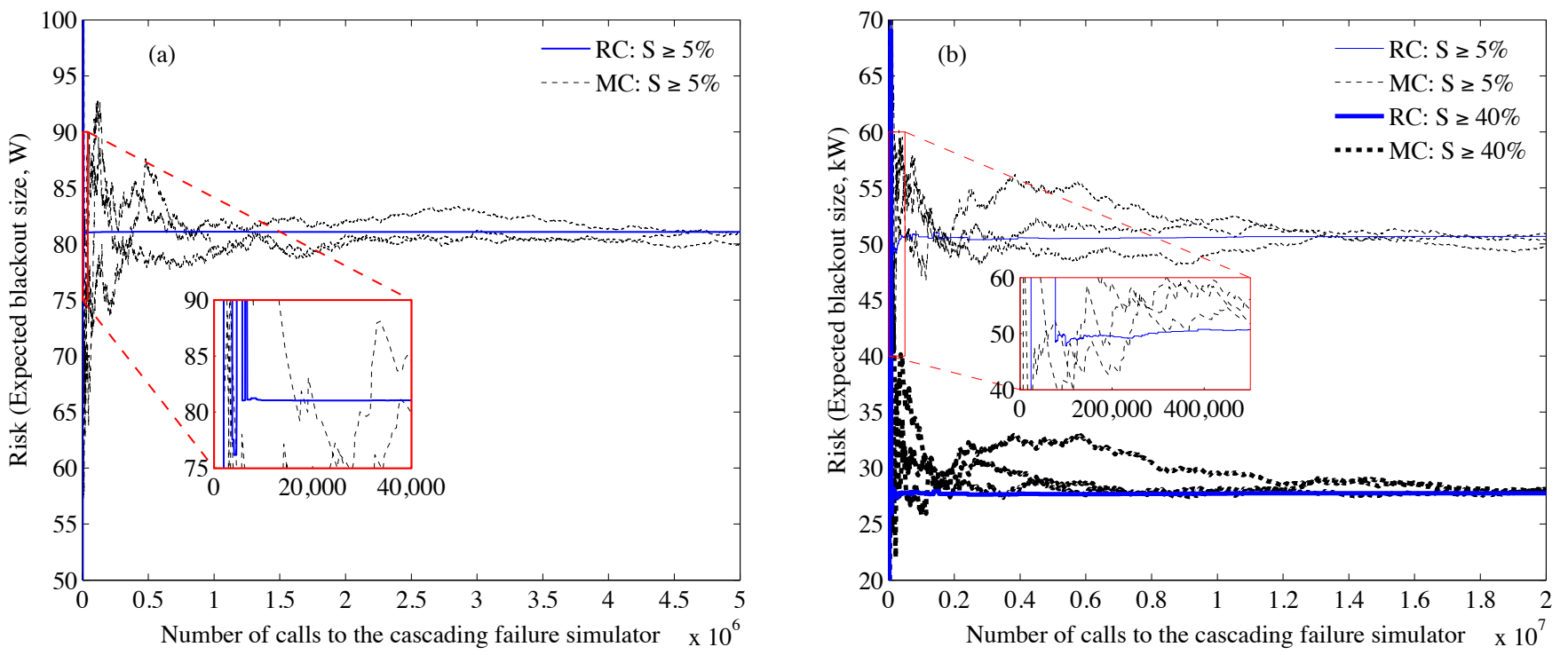

Figure 2. Cascading failure risk estimates using the Random Chemistry and Monte Carlo methods for the (a) RTS-96 and (b) Polish system, where $S$ is the blackout size. For each test case, three representative Monte Carlo runs and one Random Chemistry run (since runs for the latter are very consistent) are shown.

Table I

COMPARISON OF MONTE CARLO (MC) AND RANDOM CHEMisTRY (RC) RISK (IN KW) AFTER $10^{6}$ AND $2 \times 10^{7}$ CALLS TO THE SIMULATOR FOR 1000 BOOTSTRAPPING TRIALS

\begin{tabular}{ccccc}
\cline { 2 - 5 } & \multicolumn{2}{c}{ After $10^{6}$ calls } & \multicolumn{2}{c}{ After $2 \times 10^{7}$ calls } \\
\cline { 2 - 5 } & MC & RC & MC & RC \\
\hline Mean & 53.03 & 50.25 & 50.32 & 50.48 \\
\hline Variance & 21.87 & 0.007 & 0.95 & 0.000045 \\
\hline 95\% Confidence & $44.54-$ & $50.07-$ & $48.34-$ & $50.46-$ \\
Interval & 62.84 & 50.36 & 52.29 & 50.49 \\
\hline
\end{tabular}

\section{Investigating the components of large blackout risk}

There are a number of advantages that arise from computing risk by sampling methods such as Random Chemistry or Monte Carlo. With both methods, one can disaggregate $\hat{R}(x)$ and compute the risk that comes from blackouts of different sizes, e.g., compute the risk due to small, medium, and large blackouts, or the risk from $n-2$ and $n-3$ contingencies, although it takes much longer for Monte Carlo to find a sufficient number of outages to do this type of disaggregation. Sections IV-C1 and IV-C2 illustrate this calculation, and section IV-D shows the disaggregated (in blackout size) risk vs. load level.

A second advantage is the ability to find the sensitivity of $\hat{R}(x)$ to the failure-rates of particular transmission lines. Disaggregating $\hat{R}(x)$ and finding the most important components that impact $\hat{R}(x)$ could have tremendous value to system operators. For example, it may allow an operator to identify particular actions (such as hardening particularly vulnerable transmission lines, or reducing the load on some lines) that can substantially reduce large blackout risk.

1) Risk from blackouts of different sizes: To illustrate the separation of risk for different blackout sizes, Fig. $2 \mathrm{p}$ shows the risk for $E\left[S_{0.4}(\mathcal{C}, x)\right]$, i.e. for blackouts $40 \%$ or larger. Disaggregating risk by blackout size is particularly useful if one wants to know the impact of potential system changes, such as the addition of a new transmission line, or changes in load level. Understanding how these changes impact the risk of blackouts of different sizes should allow for more informed decision making. We study the problem of how risk changes with load in section IV-D

2) Risk from $n-2$ and $n-3$ contingencies: It is noteworthy that the majority of the risk shown in Fig. 2 comes from $n-2$ malignancies. Because of the smaller number of $n-2$ malignancies relative to $n-3$ malignancies, the Random Chemistry $n-2$ risk estimate converges much faster than the $n-3$ risk estimate. However, well after the $n-2$ risk has converged, some small fluctuations in total risk remain, due to changes in the minimal $n-3$ set size estimation (see Fig. 11p).

In order to further understand the impact of $n-3$ contingencies on blackout risk, we further explored the details of contingency sets found for the Polish system. The $n-3$ risk is composed of two portions; a small part of the risk is due to $n-3$ malignancies (herein referred to as minimal $n-3$ risk) with the remainder coming from non-minimal $n-3$ contingencies that are supersets of $n-2$ malignancies. To find the latter portion, we post-processed the data to compute the probabilities of all $n-3$ supersets of $n-2$ malignancies, using the associated $n-2$ blackout size based on Assumption 1, to avoid simulating all the supersets. The results were similar (see Table III) to what Monte Carlo finds when computing risk by sampling from both minimal and non-minimal contingencies.

Table II shows the $n-2$ and $n-3$ estimates of risk by both Monte Carlo and Random Chemistry methods for the Polish system. Although the Monte Carlo risk estimates are similar to those from Random Chemistry, Monte Carlo identifies far fewer minimal $n-3$ than non-minimal $n-3$ contingencies, thus providing much less information about the minimal $n-3$ risk. Specifically, Random Chemistry found $540 n-2$ and 38,212n-3 malignancies, whereas Monte Carlo found only 

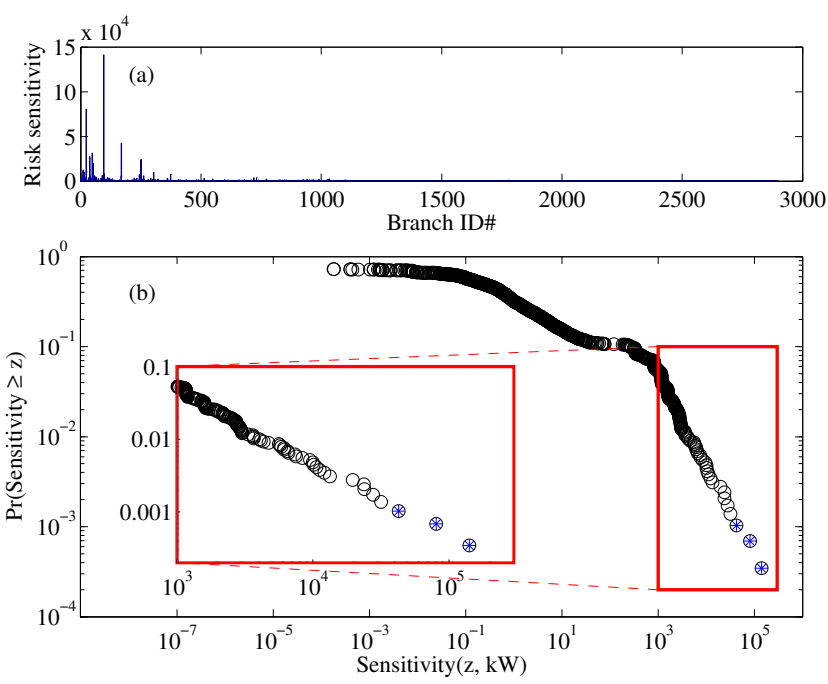

Figure 3. (a) Sensitivity of risk to branch failure probability for all 2896 branches in the Polish system, (b) Complementary Cumulative Distribution Function of sensitivities (one circle per branch); inset shows data for the 5\% of branches with the largest sensitivities. The three lines with the highest sensitivities are marked with an asterisk.

$440 n-2,41$ minimal $n-3$, and 830 non-minimal $n-$ 3 contingencies. Note that, as Table II shows, $96.7 \%$ of the Random Chemistry $n-3$ risk estimate $(11.7 \mathrm{~kW})$ was actually attributable to $n-2$ malignancies, with only $3.3 \%(0.4 \mathrm{~kW})$ due to $n-3$ malignancies.

3) Risk Sensitivity: Fig. 3 shows the sensitivity of risk to individual branch outage probabilities (derived from their failure rates) in the Polish system, as computed from (14).

Clearly, the sensitivity data in Fig. 3 show a very heavy tail; a very small number of components affect risk disproportionately. This becomes particularly clear when we look at the impact of particular lines on total risk, by computing the risk sensitivity value times the outage probability. For example, given the risk sensitivity of $1.41 \times 10^{5} \mathrm{~kW}$ and the outage probability of $1.06 \times 10^{-4}$ for branch 96 (which has the highest sensitivity value), and total risk of $50.5 \mathrm{~kW}$ in the Polish system (Fig. 2), we find that this branch can adjust $29 \%$ of overall cascading failure risk by modifying its failure probability.

These sensitivity values can be used to identify potential risk-mitigation strategies. For example, transmission lines that have a higher overall risk sensitivity factor might be targeted for increased vegetation management or improved fault detection relaying systems, which may reduce the associated lineoutage probabilities. To illustrate this approach, we identified the three branches with the highest sensitivity values, namely branches 96, 23 and 169. After identifying the branches, we

Table II

ESTIMATED $n-2$ AND $n-3$ RISK IN THE POLISH SYSTEM.

\begin{tabular}{|c|c|ccc|}
\cline { 2 - 5 } \multicolumn{1}{c|}{} & MC risk & RC risk & \multicolumn{2}{c|}{ Percent of RC risk due to } \\
\cline { 3 - 5 } \multicolumn{1}{c|}{} & $(\mathrm{kW})$ & $(\mathrm{kW})$ & $n-2$ minimal & $n-3$ minimal \\
\hline$n-2$ & 37.1 & 36.4 & $100 \%$ & NA \\
\hline$n-3$ & 11.5 & 12.1 & $96.7 \%$ & $3.3 \%$ \\
\hline Total $^{*}$ & 50.3 & 50.5 & $99.2 \%$ & $0.8 \%$ \\
\hline
\end{tabular}

reduced the three failure probabilities $\left(p_{96}, p_{23}\right.$ and $\left.p_{169}\right)$ by half, and re-computed risk. This resulted in a $33 \%$ reduction in total cascading failure risk.

\section{Estimating risk as a function of load}

Finally, in order to illustrate the potential of this approach to perform more in-depth studies of cascading failure risk, we used the Random Chemistry method to examine how cascading failure risk changes with different load levels and generator dispatch methods. To do so, we prepared $n-1$ secure versions of both systems for a range of load levels from $50 \%$ to $119 \%$ for RTS-96 and $50 \%$ to $115 \%$ for the Polish system. $119 \%$ was the highest load level at which SCDCOPF could find a solution without load shedding in the pre-contingency RTS-96 case. For the Polish system, load could increase up to $110 \%$ before a small amount of load shedding was needed to find a secure solution. For cases from $111 \%$ to $115 \%$ load, less than $1 \%$ load shedding was required to achieve $n-1$ security. For comparison purposes, we extended our study of the Polish system up to $115 \%$ load.

Fig. 4 shows risk in the RTS-96 system for all load levels for two different pre-contingency dispatch conditions. To make the analysis more useful, the risk associated with different blackout sizes are separately presented. Panel (a) shows the results produced from a SCDCOPF dispatch at each load level. In order to smooth out differences at adjacent points, each point on the graph shows the rolling average of risk across three consecutive integer percentage load levels (i.e., the datum at $90 \%$ load is the average for $89 \%, 90 \%$, and 91\%).

It is interesting to note that very-large blackout risk is greatest at about $70 \%$ load level, and decreases significantly as the load increases beyond this level. The initial increase in risk is similar to the findings in [34], [13], [20] and [30], which report a phase transition in cascading failure risk as load increases. However, depending on the dispatch method for the pre-contingency system, the risk may decrease subsequently, as Fig. 4 a shows for SCDCOPF dispatch. The later decrease in risk differs substantially from what is previously reported in the literature.

Fig. 4 p shows risk for the same load levels but with a proportional dispatch method, in which we took the $119 \%$ load case from SCDCOPF, and uniformly decreased the loads and generators to each lower load level. This dispatch method reduced risk substantially, and the relationship between load and risk now becomes monotonic. Large blackout risk is practically zero for the proportional dispatch cases. The precontingency dispatch in this case is obviously more expensive than that from the SCDCOPF, which indicates that there is an important tradeoff between generation dispatch costs and cascading failure risk.

Fig. 5 shows cascading failure risk vs. load level for the Polish system, using SCDCOPF for the pre-contingency dispatch. Again, the results suggest that that risk does not always increase with load level. In fact, risk decreases to some extent for load percentages between $100 \%$ and $110 \%$, and to a larger extent above $110 \%$ (the same cases for which some load shedding, less than $1 \%$, occurs during the pre-contingency dispatch). 

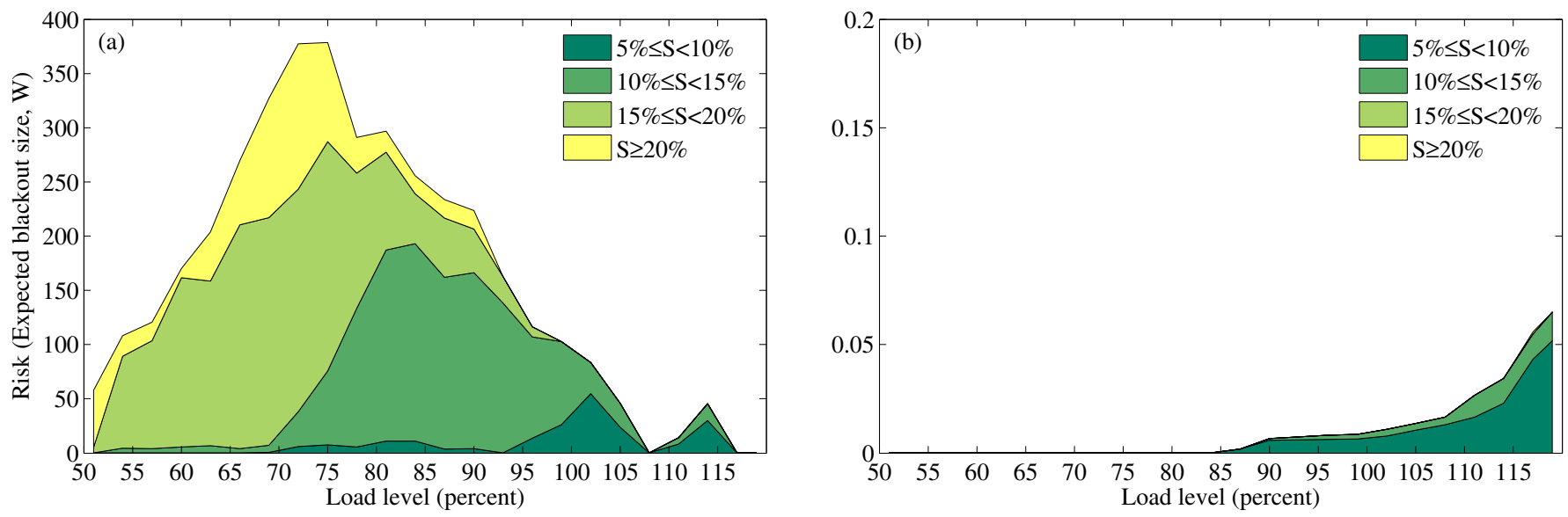

Figure 4. Cascading failure risk vs. load level for RTS-96: (a) SCDCOPF, and (b) Proportional dispatch.

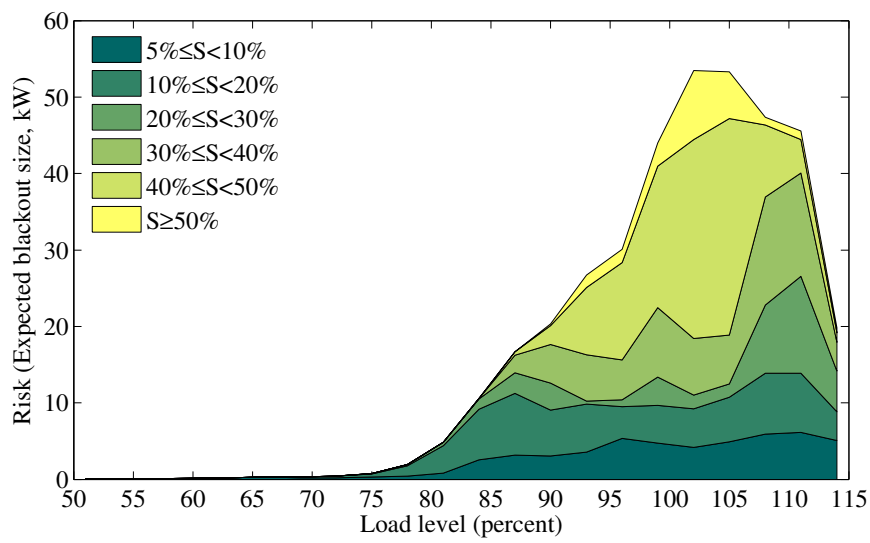

Figure 5. Cascading failure risk vs. load level for the Polish system, dispatched by SCDCOPF.

Inspection of the test systems indicates that the reduced risk at higher load levels results from the way that SCDCOPF uses more local generation with less long-distance transmission at higher load levels. In other words, at moderate load levels, the minimum cost generators are far from load centers, and important transmission corridors are loaded closer to their capacity. To illustrate this, Fig. 6 shows the total amount of power flow on the $5 \%$ most sensitive branches, found from (14), in RTS-96 and the Polish system. We observe in Fig. 6 that the total power flows on these critical lines follow the same general pattern as risk in Figs. 4 and 5 for both systems.

\section{CONCLusion AND Future Work}

This paper presents a new computationally efficient method, based on the Random Chemistry algorithm in [25], for estimating the risk of large (e.g., $\geq 5 \%$ of system load) cascading blackouts. A comparison of this method to Monte Carlo simulation for two test cases (the IEEE RTS-96, and a model of the Polish transmission system) shows that the new approach is at least two orders of magnitude faster than Monte Carlo, and does not introduce measurable bias into the estimate. The computational efficiency of the Random Chemistry approach

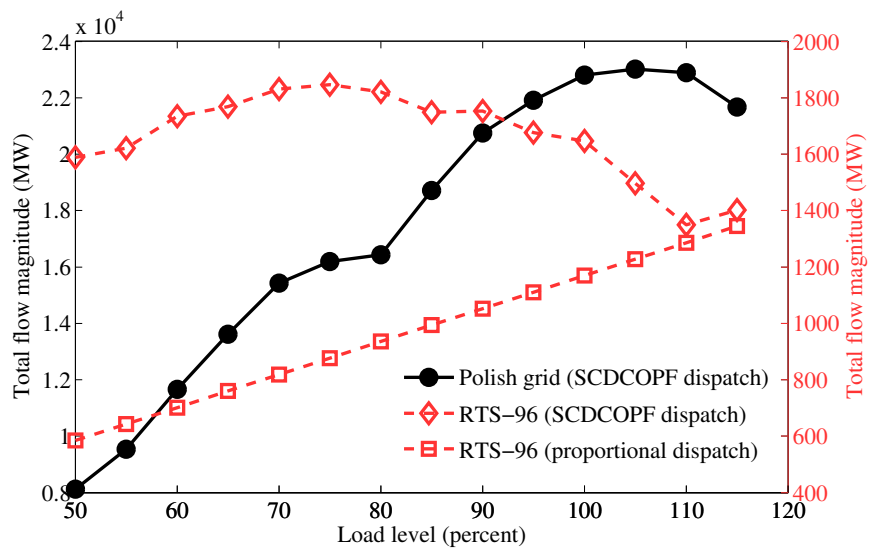

Figure 6. Total power flow magnitude on the 5\% most sensitive branches in the Polish system (solid circles, left axis) and RTS-96 (open symbols, right axis).

comes from the way that it directly searches for large blackoutcausing contingencies, as opposed to Monte Carlo, which samples broadly from all possible contingencies.

We disaggregated risk with respect to the resulting blackout sizes and the sensitivity of risk to individual branch outage probabilities in the initiating events. For the latter case, we derived a method to use the data generated by the Random Chemistry approach to quickly estimate the impact of component outage probabilities on the overall risk. For the Polish system, the results indicate that reducing the unplanned outage probabilities on three transmission lines (e.g., by more aggressive vegetation management) would produce a $33 \%$ reduction in overall cascading failure risk.

In order to illustrate the utility of the proposed approach, we computed how the risk of blackouts of various sizes changes with load level. Surprisingly, the results indicate that risk can sometimes decrease with increasing load, e.g., if generators are dispatched according to a preventative security constrained optimal power flow. However, if generators are dispatched proportionally (all generations and loads change in equal ratios with respect to a load level with low risk), blackout risk is much smaller and risk increases monotonically with load. This 
illustrates an important tradeoff between economic efficiency and blackout risk.

These results suggest a number of important topics for future research. First, the results presented here rely on the assumption that branch outages are uncorrelated and come from a relatively simple cascading failure simulator. Future work will seek to confirm that the efficiency gains associated with the Random Chemistry approach persist after modeling correlated outages and additional mechanisms of cascading. In addition, we studied only the sensitivity of risk to initiating events; studying the sensitivity of risk to events during a cascade remains for future work. Finally, future research is needed to transform the data that result from Random Chemistry into effective strategies for reducing blackout risk.

\section{APPENDIX}

\section{Summary of the RANDOM Chemistry Algorithm}

The Random Chemistry algorithm is a stochastic set-size reduction search strategy that can be used to efficiently (in logarithmic time) find minimal subsets that are associated with a certain outcome of interest. The algorithm was applied to genomic data mining in [35], and was adapted to the problem of finding $n-k$ blackout-initiating contingencies in power grids [25]. In summary, the Random Chemistry algorithm operates as follows. Initially, we use the cascading failure simulator from [25] (DCSIMSEP [33]) to find a large multiple contingency $c$ (an $n-k_{\text {init }}$ contingency, where $k_{\text {init }} \geq 40$ ) that results in a large blackout. Because $k_{\text {init }}$ is so large, this step typically requires very few tries. Then, the algorithm stochastically reduces $c$ according to a logarithmically decreasing set size reduction schedule (in this work we used $\left\{k_{\text {init }}=80\right.$, $\left.k_{2}=40, k_{3}=20, k_{4}=14, k_{5}=10, k_{6}=7, k_{\text {final }}=5\right\}$ ) by testing random subsets of the desired size until one is found that causes a large blackout. If no such subset is found within a pre-specified maximum number of tries $T$ (we used $T=20$ ), the run is restarted from a new random $n-k_{\text {init }}$ contingency. The remaining set of size $k_{\text {final }}=5$ is exhaustively searched (starting from $k=2$ ) until a minimal $n-k$ blackout-causing contingency $\left(2 \leq k \leq k_{\text {final }}\right)$ is identified. This cycle can then be repeated to obtain large collections of $n-k$ minimal hazardous contingencies.

\section{EXPLORING ASSUMPTION 1}

In this section, we test Assumption 1 for the Polish system by comparing the actual blackout sizes of all $n-3$ supersets of all $n-2$ malignancies with the estimated values, i.e., the blackout size of each $n-2$ subset. For each $n-2$ malignancy, there are $n-2=2894$ supersets that were simulated with DCSIMSEP for this comparison. We then computed the percent deviation of each $n-3$ blackout size from its malignancy subset:

$$
\epsilon=\frac{S_{a c t}-S_{e s t}}{S_{\text {est }}} \times 100
$$

where $S_{a c t}$ is the actual blackout size of an $n-3$ superset, and $S_{\text {est }}$ is the blackout size of its $n-2$ subset. Fig. 7 shows the proportional frequency of these deviations. We found that

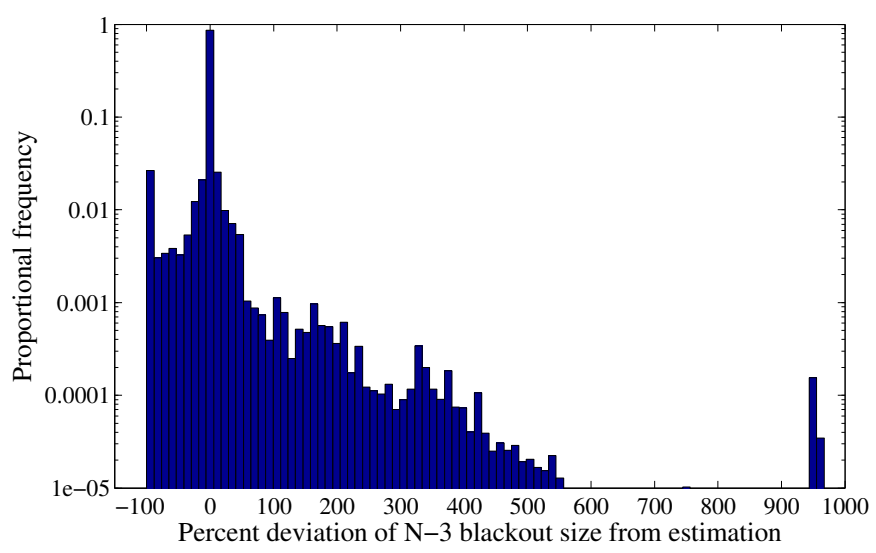

Figure 7. Proportional frequency of percent difference in actual minus estimated blackout sizes caused by non-minimal $n-3$ contingencies.

these deviations are generally in the range of $-1.9 \%\left(10^{t h}\right.$ percentile) to $0.9 \%\left(90^{\text {th }}\right.$ percentile). Ultimately, Assumption 1 resulted in the estimated $n-3$ blackout risk to be $2.5 \%$ higher than the exact value obtained by using the actual $n-3$ blackout sizes.

Note that Assumption 1 provides a weaker estimation when larger than one-extra-element supersets of malignancies are considered. However, with larger supersets, the contingencies become highly improbable, and make a negligible contribution to the total risk. Thus, we only need Assumption 1 to work for supersets with few additional elements added to a malignancy. This partially explains why the above assumption does not measurably change the Random Chemistry risk estimation results in section IV] relative to what is computed by Monte Carlo.

\section{ACKNOWLEDGMENT}

The authors gratefully acknowledge helpful conversations with Ian Dobson about these results, and Mark Wagy for assistance with an earlier version of this method.

\section{REFERENCES}

[1] Final report on the August 14, 2003 blackout in the United States and Canada. Technical report, US-Canada Power System Outage Task Force, 2004.

[2] Arizona-Southern California outages on September 8, 2011. Technical report, FERC and NERC, 2012.

[3] NERC Standard TOP-004-2. Transmission Operations, 2007.

[4] M. M. Vaiman, et al. Risk assessment of cascading outages: Methodologies and challenges. IEEE Transactions on Power Systems, 27(2):631641, 2012.

[5] R. N. Allan and R. Billinton. Reliability Evaluation of Power Systems. Plenum Press, 1996.

[6] C.L.T. Borges et al. Composite reliability evaluation by sequential Monte Carlo simulation on parallel and distributed processing environments. IEEE Transactions on Power Systems, 16(2):203-209, 2001.

[7] R. Billinton and A. Jonnavithula. Composite system adequacy assessment using sequential Monte Carlo simulation with variance reduction techniques. IEE Proceedings-Generation, Transmission and Distribution, 144(1):1-6, 1997.

[8] C. Singh and J. Mitra. Composite system reliability evaluation using state space pruning. IEEE Transactions on Power Systems, 12(1):471479, 1997.

[9] A.M. Leite da Silva, et al. Pseudo-chronological simulation for composite reliability analysis with time varying loads. IEEE Transactions on Power Systems, 15(1):73-80, 2000. 
[10] A.M. Leite da Silva, et al. Composite reliability assessment based on Monte Carlo simulation and Artificial Neural Networks. IEEE Transactions on Power Systems, 22(3):1202-1209, 2007.

[11] P. Henneaux, P.-E. Labeau, and J.-C. Maun. Blackout probabilistic risk assessment and thermal effects: Impacts of changes in generation. IEEE Transactions on Power Systems, 28(4):4722-4731, Nov 2013.

[12] B. A. Carreras et al. Initial evidence for self-organized criticality in electric power system blackouts. In Proceedings of Hawaii International Conference on System Sciences, 2000.

[13] B. A. Carreras et al. Critical points and transitions in an electric power transmission model for cascading failure blackouts. Chaos: An interdisciplinary journal of non-linear science, 12(4):985-994, 2002.

[14] I. Dobson, B.A. Carreras, and D.E. Newman. How many occurrences of rare blackout events are needed to estimate event probability? IEEE Transactions on Power Systems, 28(3):3509-3510, Aug 2013.

[15] R. Pfitzner, Konstantin Turitsyn, and Michael Chertkov. Statistical classification of cascading failures in power grids. In IEEE Power and Energy Society General Meeting, pages 1-8, July 2011.

[16] Junjian Qi, Shengwei Mei, and Feng Liu. Blackout model considering slow process. IEEE Transactions on Power Systems, 28(3):3274-3282, Aug 2013.

[17] M. Rahnamay-Naeini, et al. Stochastic analysis of cascading-failure dynamics in power grids, 2014

[18] O. Alizadeh Mousavi, R. Cherkaoui, and M. Bozorg. Blackouts risk evaluation by Monte Carlo simulation regarding cascading outages and system frequency deviation. Electric Power Systems Research, 89(0):157 - 164, 2012.

[19] D.S. Kirschen et al. A probabilistic indicator of system stress. IEEE Transactions on Power Systems, 19(3):1650-1657, 2004.

[20] J. Chen, J. S. Thorp, and I. Dobson. Cascading dynamics and mitigation assessment in power system disturbances via a hidden failure model. International Journal of Electrical Power \& Energy Systems, 27(4):318326, 2005.

[21] J. Kim, J.A. Bucklew, and I. Dobson. Splitting method for speedy simulation of cascading blackouts. IEEE Transactions on Power Systems, 28(3):3010-3017, 2013

[22] Q. Chen and L. Mili. Composite power system vulnerability evaluation to cascading failures using importance sampling and antithetic variates. IEEE Transactions on Power Systems, 28(3):2321-2330, 2013.

[23] Hui Ren and Ian Dobson. Using transmission line outage data to estimate cascading failure propagation in an electric power system. IEEE Transactions on Circuits and Systems-II: Express Briefs, 55(9):927-931, 2008.

[24] I. Dobson. Estimating the propagation and extent of cascading line outages from utility data with a branching process. IEEE Transactions on Power Systems, 27(4):2146-2155, 2012.

[25] M.J. Eppstein and P.D.H. Hines. A "Random Chemistry" algorithm for identifying collections of multiple contingencies that initiate cascading failure. IEEE Transactions on Power Systems, 27(3):1698-1705, 2012.

[26] P. Rezaei, P.D.H. Hines, and M.J. Eppstein. Estimating cascading failure risk: Comparing Monte Carlo sampling and random chemistry. In IEEE Power and Energy Society General Meeting, pages 1-5, July 2014.

[27] P. Rezaei and P.D.H. Hines. Changes in cascading failure risk with generator dispatch method and system load level. In IEEE PES Transmission and Distribution Conference and Exposition, pages 1-5, 2014.

[28] W. G. Cochran. Sampling Techniques. Wiley, 2nd edition, 1963.

[29] C. Grigg, et al. The IEEE reliability test system-1996. a report prepared by the reliability test system task force of the application of probability methods subcommittee. IEEE Transactions on Power Systems, 14(3):1010-1020, 1999.

[30] D.P. Nedic, et al. Criticality in a cascading failure blackout model International Journal of Electrical Power and Energy Systems, 28(9):627 - 633, 2006.

[31] R.D. Zimmerman, C.E. Murillo-Sánchez, and R.J. Thomas. MATPOWER: Steady-state operations, planning, and analysis tools for power systems research and education. IEEE Transactions on Power Systems, 26(1):12 -19, feb. 2011.

[32] http://www.eia.gov/.

[33] P.D.H. Hines. DCSIMSEP: A simulator of cascading separation in power grids. http://uvm.edu/ phines/dcsimsep/

[34] H. Liao, J. Apt, and S. Talukdar. Phase transitions in the probability of cascading failures. In Electricity Transmission in Deregulated Markets: Conference at Carnegie Mellon University, Pittsburgh, PA, 2004.

[35] M.J. Eppstein et al. Genomic mining for complex disease traits with "Random Chemistry". Genetic Programming and Evolvable Machines, 8(4):395-411, 2007.

\section{AUTHOR BIOGRAPHIES}

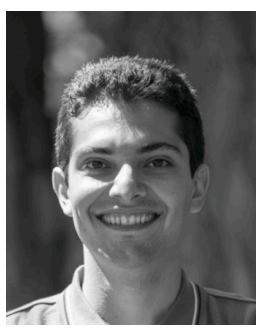

Pooya Rezaei (S'12) received the M.Sc. and B.Sc. degrees in Electrical Engineering from Sharif University of Technology (2010) and University of Tehran (2008) respectively. Currently, he is pursuing the $\mathrm{Ph} . \mathrm{D}$. in Electrical Engineering at the University of Vermont.

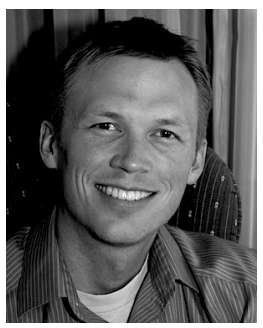

Paul D. H. Hines (S'96,M'07,SM'14) received the Ph.D. in Engineering and Public Policy from Carnegie Mellon University in 2007 and M.S. (2001) and B.S. (1997) degrees in Electrical Engineering from the University of Washington and Seattle Pacific University, respectively. $\mathrm{He}$ is currently an Associate Professor in the School of Engineering at the University of Vermont.

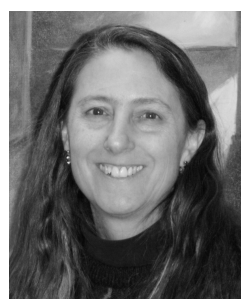

Margaret J. Eppstein is Professor and Chair of Computer Science at the University of Vermont (UVM), where she has been on the faculty since 1983. She received a B.S. in Zoology from Michigan State University in 1978, an M.S. in Computer Science from UVM in 1983, and a Ph.D. in Environmental Engineering at UVM in 1997. She was the founding director of the Vermont Complex Systems Center (2006-2010). 